\title{
Pulsar Studies at High Radio Frequencies
}

\author{
Richard Wielebinski \\ Max-Planck-Institut für Radioastronomie, Auf dem Hügel 69, 53121 \\ Bonn, Germany
}

\begin{abstract}
Pulsars were discovered at $81.5 \mathrm{MHz}$ and a lot of the studies of these exciting objects have been made up to the present time at radio frequencies below $1.6 \mathrm{GHz}$. The reasons for this concentration on the low radio frequency characteristics of pulsars is the fact that the spectra are very steep and that very few radio telescopes exist that are capable of efficient operations at high radio frequencies. The Effelsberg 100-m radio telescope of the Max-Planck-Institut für Radioastronomie operates regularly up to the frequency of $50 \mathrm{GHz}$ and was used to study pulsars at $\mathrm{cm} / \mathrm{mm}$-wavelengths. In the southern skies the Parkes $64-\mathrm{m}$ telescope has been used to study pulsars up to $8.4 \mathrm{GHz}$. One pulsar has been detected at $87 \mathrm{GHz}$ with the $30-\mathrm{m}$ Pico Veleta telescope of IRAM.

The studies of pulsars over the whole frequency range are of great importance because this is necessary for the elucidation of the mechanism that is responsible for the pulsar emission. The high polarization of pulsar radio emission at lower radio frequencies has supported the hypothesis of a coherent emission mechanism, which is required to generate the high luminosity. It has been known for some time that pulsars, unlike other radio sources, have a lower polarization at high radio frequencies. Recently a change of pulsar spectrum, a flattening or possibly an inversion has been observed at the highest radio frequencies. The inversion of the pulsar spectrum seems to coincide with a complete depolarization of some pulsars.

Millisecond pulsars are less luminous than normal pulsars. This makes them even more difficult to detect at higher radio frequencies. Recent observations have extended the spectra of ten millisecond pulsars up to $4.85 \mathrm{GHz}$. The results imply that millisecond pulsars have properties very similar to normal (slow) pulsars, which suggests similar emission mechanisms.
\end{abstract}

\section{Introduction}

Soon after the announcement of the discovery of pulsars by Hewish et al. (1968), most of the radio telescopes around the world turned to pulsar observations. Many further parameters of pulsars were established like the exact period, period derivative, pulse shape, the polarization and spectrum. Observing at five frequencies with the Parkes telescope Robinson et al. (1968) established the spectrum of CP1919 from $85 \mathrm{MHz}$ to $2.7 \mathrm{GHz}$. The spectral index was steep 
at decimeter wavelength $(\alpha=-1.8)$ showing a spectral break $(\alpha \sim-3.0)$ at the highest frequencies. This result implied that pulsar studies would be very difficult at high radio frequencies.

The next step in the high frequency observations of pulsars had to wait for the completion of the 100-m radio telescope of the MPIfR at Effelsberg where five pulsar were studied at $10.6 \mathrm{GHz}$ (Wielebinski et al. 1972). The consequent use of high sensitivity of the 100-m radio telescope at Effelsberg allowed the study of pulsars at $22 \mathrm{GHz}$ (Bartel et al. 1977; 1978) and finally at mm-wavelengths (Wielebinski et al. 1993). The final step, in the quest of detecting pulsars at the highest radio frequencies, was made by Morris et al. (1997) who detected one pulsar at $87 \mathrm{GHz}$ with the Pico Veleta telescope of IRAM.

Pulsar polarization was first detected by Lyne \& Smith (1968). The observations of the swing of the linear polarization vector by Radhakrishnan \& Cooke (1969) clinched the argument that the pulsar emission comes from the magnetic poles of a rotating neutron star. Observers who studied pulsar polarization at higher frequencies (e.g. Manchester 1971; Morris et al. 1981) came to the realization that pulsar polarization, unlike for most other radio sources, falls with increasing frequency. It has been noted by Manchester et al. (1973) that for some pulsars the linear polarization fraction is constant up to some critical frequency, but then decreases rapidly with increasing frequency. The early polarization studies have been followed up to short $\mathrm{cm}$ and $\mathrm{mm}$ wavelengths by Xilouris et al. (1996). The observations of larger samples of pulsars at high radio frequencies (e.g. von Hoensbroech \& Xilouris 1997) have contributed much to the understanding of the evolution of pulsar polarization with frequency. In particular a class of pulsars has been discovered by von Hoensbroech et al. (1998) in which the linear polarization decreases while the circular polarization increases with frequency.

Millisecond pulsars were detected by Backer et al. (1982) following a search of radio sources with very steep spectra. These objects were interpreted to be spun-up (or recycled) pulsars, where the fast period comes through a binary interaction, not as a result of recent birth. The millisecond pulsars have a rather low period derivative and have become accepted to be the best clocks. The characteristics of the millisecond pulsars have not been extensively studied so far, especially at higher radio frequencies, because they are objects with rather low radio luminosity. In a recent series of papers, originating from the $100-\mathrm{m}$ telescope, millisecond pulsars have been studied up to the frequency of $4.85 \mathrm{GHz}$ (Kramer et al. 1998, 1999; Xilouris et al. 1998).

\section{High Frequency Radio Surveys}

Information on the characteristics of pulsars at frequencies below $1.6 \mathrm{GHz}$ was catalogued by Taylor et al. (1993). A revised version of the Taylor et al. catalogue contained information on 706 pulsars at $400 \mathrm{MHz}$ and $1.4 \mathrm{GHz}$. The study of 280 pulsars by Lorimer et al. (1995) at lower frequencies included flux density information on some of the objects at $1.6 \mathrm{GHz}$. A pioneering study of a larger sample of pulsars at $2.7,4.9$ and $10.7 \mathrm{GHz}$ was made by Sieber et al. (1975). This work showed that there is a considerable evolution of pulse shape with frequency. Seiradakis et al. (1995) presented pulse shapes and tabulated 
PSR $30355+54$

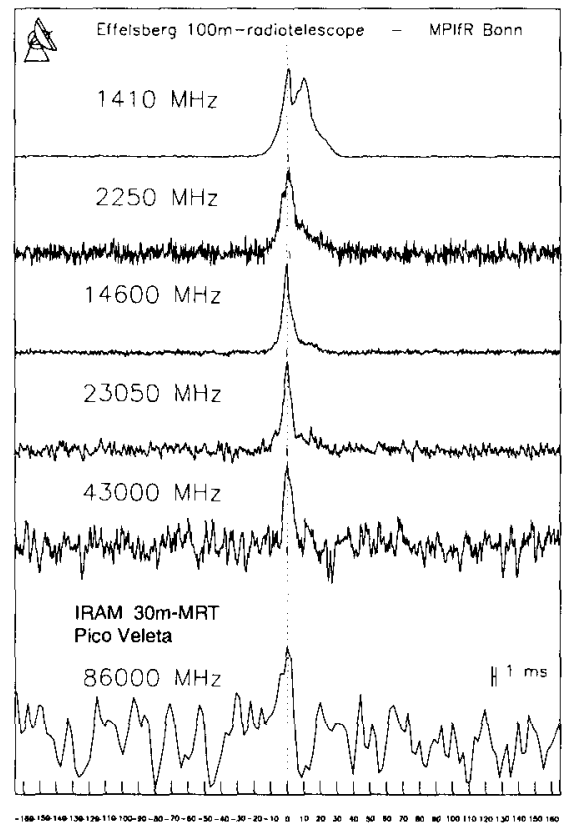

Loncitude [deal

Figure 1. Time aligned profiles of PSR $0355+54$

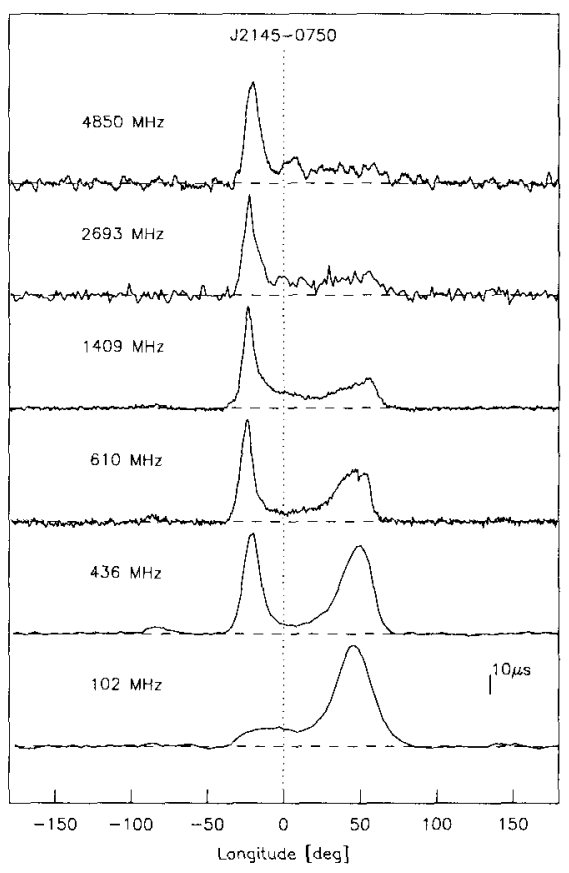

Figure 2. Pulse shape evolution for J2145-0750

flux values for 183 pulsars at $1.4 \mathrm{GHz}$, for 46 at $4.8 \mathrm{GHz}$ and for 24 at $10.6 \mathrm{GHz}$. It must be noted that the $4.8 \mathrm{GHz}$ pulse shapes catalogued by Seiradakis et al. (1995) were a result of observations made over the period 1972-1992. The advent of a new low noise HEMT amplifier at Effelsberg allowed the study of 87 weaker pulsars in only $\sim 100$ hours of observing time (Kijak et al. 1998). The large bandwidth of the new HEMT amplifiers in the frequency range 30$50 \mathrm{GHz}$ allowed the detection of pulsars at long mm-wavelengths (Wielebinski et al. 1993; Kramer et al. 1997). Finally an attempt was made to extend these observations to even shorter mm-wavelengths, using the Pico Veleta telescope of IRAM, resulting in a detection of the pulsar PSR $0355+54$ at $\lambda=3.4 \mathrm{~mm}$ and the setting of an upper flux limit for PSR $2021+51$. These short mm-wavelength observations were made with a $500 \mathrm{MHz}$ bandwidth SIS receiver system so that the present developments of new broad bandwidth MIMIC amplifiers promise a new era of pulsar studies. Also the plans for the Atacama Large MM Array (ALMA) should allow us to extend pulsar studies to sub-mm wavelength.

\section{Pulsar Spectra}

The spectrum of a pulsar is the basic information that is a reflection of the pulsar emission process. The determination of pulsar spectra is not easy, due to the various fading characteristics of pulsars. The typical spectrum of a pulsar 
has a rather steep fall-off in the decimeter wavelength range, a low frequency turnover and a high frequency break (steepening) at the shortest wavelength. An early compilation of pulsar spectra, which was presented by Sieber (1973), shows these characteristics for 27 objects. In the paper of Malofeev \& Malov (1980) 39 spectra are shown indicating that the high frequency break occurs rather often. The compilation of 45 pulsar spectra, determined over a wider frequency range by Malofeev at al. (1994), showed that $\sim 30 \%$ of the pulsars showed the high frequency break. The surprise in this field of research came as a result of the $\mathrm{mm}$-wavelength observations of Wielebinski et al. (1993) and Kramer et al. (1997). In these papers it was shown that for some pulsars the $\mathrm{mm}$-wavelength flux values are very significantly above what was expected from the extrapolations of lower frequency data. This result, which possibly suggests a new emission process, will be discussed later in conjunction with pulsar polarization. It should be noted here that the classical spectrum of the Crab pulsar (e.g. Smith 1977) shows the steep spectrum at radio frequencies, but also high flux values in the infrared and optical range, suggesting that a turn-up in the spectrum at mm- or sub-mm wavelengths is to be expected. In the proceedings of this conference several papers discuss the spectra of large samples of pulsars (e.g. see Malofeev; Maron et al.) showing the need to study well defined samples.

\section{Pulsar Polarization}

In all radio polarization measurements the general rule is that the higher the observing frequency the higher the polarization. Observations of polarized Galactic emission, polarization of radio sources (supernova remnants, normal galaxies, radio galaxies, etc.) follow this general rule. Pulsar polarization at meter wavelength was known to be high, sometimes up to $100 \%$ (e.g. Manchester 1971). It was also realized that the pulsar polarization falls (Manchester et al. 1973) to higher frequencies, but the exact depolarization evolution was not known due to lack of good high frequency data.

In a series of papers using the $100 \mathrm{~m}$ radio telescope (e.g. Morris et al. 1981; Xilouris et al. 1996) the observations of pulsar polarization were extended to $32 \mathrm{GHz}$. Also the more recent work of von Hoensbroech (1999) gave a good insight into the evolution of a larger sample of pulsars. The standard evolutionary development is that the polarization of all components falls with increasing frequency. Some pulsars show an increase in polarization of a sub-component of the pulse profile while the other component (or components) show falling polarization. In a few cases the linear polarization falls with frequency while the circular polarization increases.

An interesting observation has been made for pulsars that show a flattening (or turn up) of their spectra at high frequencies. In these objects the polarization falls to zero when the spectral change is observed. A simple interpretation of this correlation would be that coherent (polarized) emission stops and a new (incoherent) emission process takes over. This would 'fill' the gap in the spectrum of such objects as the Crab pulsar. Recent results of Moffet \& Hankins (1999) also show a spectral flattening in the Crab pulsar spectrum and support this suggestion. 


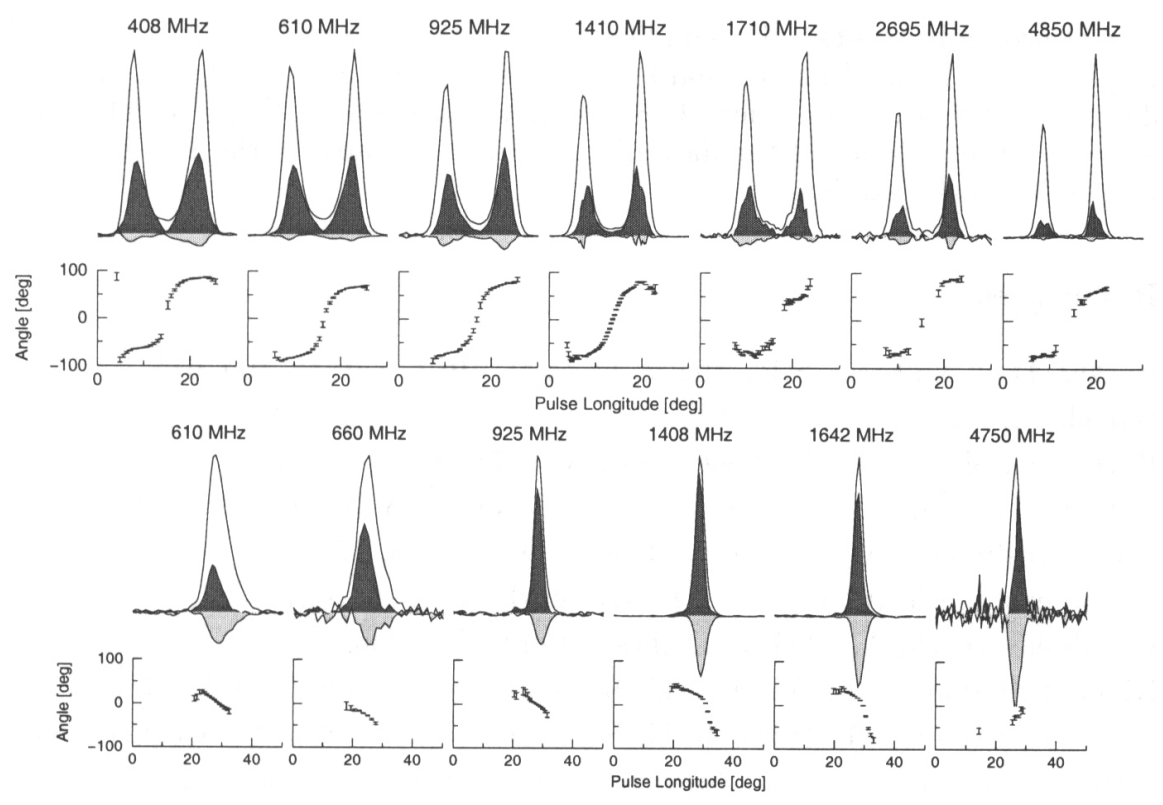

Figure 3. Pulse polarization evolution of PSR 0525+21 (above) and PSR B1737-30 (below). Circular polarization is light shaded.

\section{Millisecond Pulsars at High Frequencies}

Most of the studies of millisecond pulsars were made so far at lower radio frequencies. Recent consequent observations of a large sample of millisecond pulsars at $1.4 \mathrm{GHz}$ (Kramer et al. 1998; Xilouris et al. 1998) allowed to make significant statistical conclusions. In particular it was possible to compare the characteristics of a well defined volume sample of normal pulsars with millisecond pulsars. The general conclusion is that the two classes of objects, with so different evolutionary history, show many similar characteristics. The only one significant difference is in the luminosity of the two classes of pulsars. Millisecond pulsars have a significantly lower luminosity. Surprisingly the number of sub-pulse components, which was taken for a long time to be quite different for millisecond pulsars, shows a similar distribution when many objects are compared. The polarization vector swing for millisecond pulsars is very narrow, suggesting that the emission indeed originates close to the neutron star surface. Observations of millisecond pulsars were shown to be possible at $4.8 \mathrm{GHz}$ by Kijak et al. (1997). These studies were extended to a larger sample of millisecond pulsars by Kramer et al. (1999). The multi-frequency studies of millisecond pulsars have shown that the polarization falls to high frequencies as in normal (slow) pulsars. The spectra of millisecond pulsars show so far no spectral break. Obviously additional observations of millisecond pulsars are needed at both the highest but also at low radio frequencies. Some $102 \mathrm{MHz}$ data for millisecond pulsars were published by Kuzmin \& Losovsky (1996) and are presented in this volume (Kuzmin et al.; Malofeev et al.). 
Acknowledgments. I would like to thank the 'pulsar group' at the MPIfR. In particular, I would like to thank W. Sieber, M. Kramer, N. Wex, J.H. Seiradakis, K. Xilouris, D. Lorimer, A. Kuzmin, V. Malofeev, J. Gil, J. Kijak as well as many others. Much of the data discussed are available in the Pan-European Pulsar Network data base.

\section{References}

Backer, D.C, Kulkarni, S.R., Heiles, C. et al. 1982, Nature, 300, 615

Bartel, N., Sieber, W., \& Wielebinski, R. 1977, A\&A, 55, 319

Bartel, N., Sieber, W., \& Wielebinski, R. 1978, A\&A, 68, 361

Hewish, A., Bell, S.J., Pilkington, J.D.H. et al. 1968, Nature, 217, 709 von Hoensbroech, A., \& Xilouris, K.M. 1997, A\&AS, 126, 121

von Hoensbroech, A., Kijak, J., \& Krawczyk, A. 1998, A\&A, 334, 571

von Hoensbroech, A. 1999, PhD thesis, Bonn University

Kijak, J., Kramer, M., Wielebinski, R., \& Jessner, A. 1997, A\&A, 318, L63

Kijak, J., Kramer, M., Wielebinski, R., \& Jessner, A. 1998, A\&AS, 127, 153

Kramer, M., Xilouris, K.M., Jessner, A. et al. 1996, A\&A, 306, 867

Kramer, M, Jessner, A., Doroshenko O., \& Wielebinski, R. 1997, ApJ, 488, 364

Kramer, M., Xilouris, K.M., Lorimer, D.R. et al. 1998, ApJ, 501, 270

Kramer, M., Lange, C., Lorimer, D.R. et al. 1999, ApJ (in press)

Kuzmin, A.D, \& Losovsky, B.Ya. 1996, A\&A, 308, 91

Lorimer, D.R, Yates, J.A., Lyne, A.G., \& Gould D.M. 1995, MNRAS, 273, 411

Lyne, A.G., \& Smith, F.G. 1968, Na.ture, 218, 124

Malofeev, V., \& Malov, I. 1980, Soviet Ast., 24 (10), 54

Malofeev, V., Gil, J.A., Jessner, A. et al. 1994, A\&A, 285, 201

Manchester, R.N. 1971, ApJS, 23, 283

Manchester, R.N., Taylor, J.H., \& Huguenin, G.R. 1973, ApJ, 179, L7

Moffet, D.A, \& Hankins, T.H. 1999, ApJ, 522, 1046

Morris, D.A., Graham, D.A., Sieber, W. et al. 1981, A\&AS, 46, 421

Morris, D.A., Kramer, M., Thum, C. et al. 1997, A\&A, 322, L17

Radhakrishnan, V., \& Cooke, D.J. 1969, Astrophys. Lett., 3, 225

Robinson, B.J., Cooper, B.F.C., Gardner, F.F. et al. 1968, Nature, 218, 1143

Seiradakis, J.H., Gil, J., Graham, D.A. et al. 1995, A\&AS, 111, 205

Sieber, W. 1973, A\&A, 28, 237

Sieber, W., Reinecke, R., \& Wielebinski, R. 1975, A\&A, 38, 169

Smith, F.G. 1977 in Pulsars (Cambridge: Cambridge University Press), 118

Taylor, J.H., Manchester, R.N, \& Lyne A.G. 1993, ApJS, 88, 529

Wielebinski, R., Sieber, W., Graham, D.A. et al. 1972, Nature, 240, 131

Wielebinski, R., Jessner, A., Kramer, M., \& Gil, J. 1993, A\&A, 272, L13

Xilouris, K.M., Kramer, M., Jessner, A. et al. 1996, A\&A, 309, 481

Xilouris, K.M., Kramer, M., Jessner, A. et al. 1998, ApJ, 501, 286 